\title{
Molecular evolution and adaptation of the mitochondrial cytochrome $b$ gene in the subgenus Martes
}

\author{
B. Li ${ }^{1,2}$, B. Malyarchuk ${ }^{3}$, X.B. He $\mathrm{He}^{1,4}$ and M. Derenko ${ }^{3}$ \\ ${ }^{1}$ Northeast Forestry University, College of Wildlife Resources, Harbin, China \\ ${ }^{2}$ State Forestry Administration Detecting Center of Wildlife Resources, \\ Harbin, China \\ ${ }^{3}$ Genetics Laboratory, Institute of Biological Problems of the North, \\ Far-East Branch of Russian Academy of Sciences, Magadan, Russia \\ ${ }^{4}$ Harbin Zoo, Harbin, China
}

Corresponding author: B. Malyarchuk

E-mail: malyarchuk@ibpn.ru

Genet. Mol. Res. 12 (3): 3944-3954 (2013)

Received January 9, 2013

Accepted May 23, 2013

Published September 23, 2013

DOI http://dx.doi.org/10.4238/2013.September.23.13

\begin{abstract}
Martes species represent a typical example of rapid evolutionary radiation and a recent speciation event. To identify regions of the genome that experienced adaptive evolution, which might provide clues to their functional importance and may be informative about the features that make each species unique, we sought evidence of molecular adaptation in the mitochondrial DNA (mtDNA) cytochrome $b$ gene in the subgenus Martes. Complete sequences of the cytochrome $b$ gene were obtained from 87 samples, including 49 sables, 28 pine martens, and 10 stone martens, and were combined with mtDNA sequences of other true martens, such as $M$. melampus and $M$. americana. Analysis of the cytochrome $b$ gene variation in true martens has shown that the evolution of this gene is under negative selection. In contrast, positive selection on the cytochrome $b$ protein has been detected by means of the software TreeSAAP using a phylogenetic reconstruction of Martes
\end{abstract}


taxa. Signatures of adaptive variation in cytochrome $b$ were restricted to the transmembrane domains, which likely function as proton pumps. We compared results of different methods for testing selection and molecular adaptation, and we supposed that the radical changes of the cytochrome $b$ amino acid residues in the subgenus Martes may be the result of molecular adaptation to specific environmental conditions coupled with species dispersals.

Key words: Subgenus Martes; Adaptive evolution; Mitochondrial DNA; Cytochrome $b$

\section{INTRODUCTION}

Recently, the search for evidence of adaptive evolution at the molecular level has been at the forefront of genetics research. A principal motivation has been to identify regions of the genome that have experienced adaptive evolution because this might provide clues to their functional importance and may be informative about the features that make each species unique. The mitochondrial cytochrome $b$ gene is one of the genes encoding an integral membrane protein component of the cytochrome $b c 1$ complex, which catalyzes the redox transfer of electrons from ubiquinone to cytochrome $c$ in the mitochondrial electron transport chain (Trumpower, 1990). Because the efficiency of the electron transport chain governs key aspects of aerobic energy metabolism, several investigations have suggested that functional modifications of cytochrome $b$ may be involved in physiological adaptations to different thermal environments (Gershoni et al., 2009).

One of the ways to detect the adaptive evolution of protein-coding genes is to calculate the positive selection that leads to radical structural or functional shifts in local regions of the protein. For example, McClellan et al. (2005) suggested an estimate of positive-destabilizing selection that represented the unambiguous signature of molecular adaptation because when radical amino acid changes are favored by selection, they result in local directional shifts in biochemical function, structure, or both (McClellan et al., 2005). Using these methods, many pioneer studies suggested that mutations occurring in mammalian cytochrome $b$ may have functional implications in species with peculiar metabolic requirements, such as adaptation to the low energy diet or large body size of elephants, adaptation to extreme $\mathrm{O}_{2}$ requirements in cetaceans, and adaptation to high altitudes among alpacas (McClellan and McCracken, 2001; Fink et al., 2004; McClellan et al., 2005; da Fonseca et al., 2008; Foote et al., 2011).

Here, we devoted special attention to Martes species because they represent a typical example of rapid evolutionary radiation and a recent speciation event (Yu et al., 2011). The genus Martes has 8 extant species (Anderson, 1970). Three subgenera of Martes are recognized: Pekania Gray, fisher (M. pennanti); Charronia Gray, yellow-throated marten (M. flavigula) and Nilgiri marten (M. gwatkinsii); and Martes Pinel, true martens (sable M. zibellina, pine marten M. martes, Japanese marten M. melampus, American marten M. americana, and stone marten M. foina) (Stone and Cook, 2002). In the distribution of marten species, the pine marten is mainly present in Western Europe. The sable replaces pine marten at some point east of the Ural Mountains and has a widespread geographic distribution, which is replaced by the yellow-throated marten in East Asia, by the Nilgiri marten in southern India, and by the Japa- 
nese marten in southern Japan. The stone marten is distributed throughout Eurasia. In North America, 2 species are present, the fisher and American marten.

Because some Martes species are adapted to particular ranges with boreal latitudes, they may be subject to local adaptations of metabolism and energy as previously documented by several investigations of the mitochondrial genome in different vertebrates (McClellan et al., 2005; da Fonseca et al., 2008; Malyarchuk et al., 2010; Foote et al., 2011). However, there are no studies that revealed the molecular adaptation of the mitochondrial cytochrome $b$ gene in Martes. In our analysis, we sought evidence of adaptive evolution in the cytochrome $b$ gene of the sables from different geographic populations as well as among the pine martens, stone martens, and other species of true martens using several analytical approaches to detect positive selection.

\section{MATERIAL AND METHODS}

\section{Sequence data}

We examined 87 samples from 8 different regions of Eurasia, including 49 sables, 28 pine martens, and 10 stone martens (Table 1). Genomic DNA was isolated from various tissues by the standard phenol-chloroform method. The complete cytochrome $b$ gene was amplified by polymerase chain reaction (PCR) with the upstream primer Mz-L1 (Li et al., 2011) and the downstream primer H15915 (Irwin et al., 1991).

Table 1. Cytochrome $b$ gene haplotypes and sampling localities of specimens examined in the present study.

\begin{tabular}{|c|c|c|c|}
\hline Species & Source & Haplotypes or accession No. & References \\
\hline \multirow[t]{8}{*}{ Martes zibellina } & Xiaoxing'anling Mountains, China & $\mathrm{Mz1}, \mathrm{Mz} 2, \mathrm{Mz} 3$ & Present study; Li et al., 2013 \\
\hline & Daxing'anling Mountains, China & Mz4, Mz5, Mz6 & Present study; Li et al., 2013 \\
\hline & China (?) & Mz7, Mz8 & Present study; Li et al., 2013 \\
\hline & Arhara River, Amur region, Russia & $\mathrm{Mz} 1, \mathrm{Mz} 9$ & Present study; Li et al., 2013 \\
\hline & Lazo raion, Russia & $\begin{array}{l}\text { Mz4, Mz10(2), Mz11, Mz12(2), Mz14(2), } \\
\text { Mz15, Mz16(4), Mz17(4), Mz18(2), Mz19 }\end{array}$ & Present study; Li et al., 2013 \\
\hline & Verkhnebureinsk raion, Russia & $\begin{array}{l}\operatorname{Mz1}, \operatorname{Mz} 5(2), \operatorname{Mz} 7(3), \operatorname{Mz16}, \operatorname{Mz} 20 \\
\text { Mz21(3), Mz22, Mz23(3) }\end{array}$ & Present study; Li et al., 2013 \\
\hline & Russia (?) & Mz13, Mz15, Mz24, Mz25 & Present study; Li et al., 2013 \\
\hline & Hokkaido, Japan & $\mathrm{AB} 012356-\mathrm{AB} 012360$ & Kurose et al., 1999 \\
\hline \multirow[t]{4}{*}{ M. martes } & Jutland and Zealand, Denmark & $\operatorname{Mm} 1(9), \operatorname{Mm} 2(12), M m 3$ & Present study \\
\hline & Southern Sweden & $\operatorname{Mm} 1$ (3), Mm3, Mm4 (2) & Present study \\
\hline & University of Alaska Museum & AF448241 & Stone and Cook, 2002 \\
\hline & Moscow region, Russia & AB051237 & Hosoda et al., 2000 \\
\hline M. melampus & Southern Japan* & $\mathrm{AB} 012341-\mathrm{AB} 012355$ & Kurose et al., 1999 \\
\hline M. americana & USA* & $\begin{array}{l}\text { AY121275, AY121193, AB051234, AF154964, } \\
\text { AF154968, AF268273, AF448237, AF448238 }\end{array}$ & Stone et al., 2002 \\
\hline \multirow[t]{2}{*}{ M. foina } & Jutland, Denmark & $\operatorname{Mf1}(4), \operatorname{Mf2}(3), \operatorname{Mf3}(3)$ & Present study \\
\hline & Kunming, China & AB051236 & Hosoda et al., 2000 \\
\hline M. flavigula & Duzit Zoo, Thailand & AB012362 & Kurose et al., 1999 \\
\hline Gulo gulo & Sakhalin, Russia & AB051245 & Hosoda et al., 2000 \\
\hline M. pennanti & Massachusetts, USA & AF057131 & Koepfli and Wayne, 1998 \\
\hline Mustela vison & Brookfield Zoo, USA & AF057129 & Koepfli and Wayne, 1998 \\
\hline
\end{tabular}

Number of specimens with designated haplotype is indicated in parentheses. Question-mark denotes that exact geographic origin of the specimen in Russia or in China is unclear. Asterisks denote that detailed geographic origin of haplotypes can be found in references.

A 50- $\mu$ L PCR mix contained about 100 ng DNA template, 1 X PCR buffer $(10 \mathrm{mM}$ 
Tris- $\mathrm{HCl} \mathrm{pH} 8.3,50 \mathrm{mM} \mathrm{KCl}$, and $1.5 \mathrm{mM} \mathrm{MgCl}$ ), $0.2 \mathrm{mM}$ dNTP, $0.2 \mu \mathrm{M}$ each primer, and 2.5 units TaqTM DNA polymerase (TaKaRa Biotechnology Dalian Co., China). Amplifications were carried out on a GeneAmp PCR System 9700 (Applied Biosystems, Foster City, CA, USA) under the following reaction conditions: $94^{\circ} \mathrm{C}$ for $3 \mathrm{~min} ; 35$ cycles of $94^{\circ} \mathrm{C}$ for 30 $\mathrm{s}, 50^{\circ} \mathrm{C}$ for $30 \mathrm{~s}$, and $72^{\circ} \mathrm{C}$ for $1 \mathrm{~min}$; and a final extension of $5 \mathrm{~min}$ at $72^{\circ} \mathrm{C}$.

PCR products were recovered and purified using an AxyPrep ${ }^{\mathrm{TM}}$ DNA Gel Extraction Kit (AxyGen Biosciences, Hangzhou, China) according to the manufacturer instructions. Recovered PCR products were sequenced directly using the same primers on an ABI 3730 DNA Analyzer (performed by BGI, Beijing, China). Consensus sequences were generated using the SeqMan software (DNAStar Inc., Madison, WI, USA).

Nucleotide sequences of the entire cytochrome $b$ gene of the sables, the pine martens, and the stone martens were deposited in GenBank. In addition, some previously published complete cytochrome $b$ gene sequences were also used for analysis of molecular data (Table 1 ). We did not find any cytochrome $b$ gene sequences of the Nilgiri marten in GenBank, so this taxon was excluded from further analyses.

\section{Alignments and sequence characterizations}

Nucleotide sequences of the cytochrome $b$ gene were aligned using the ClustalW algorithm of the program MEGA 5.05 (Tamura et al., 2011). Molecular diversity indices, such as haplotype diversity $(h)$, nucleotide diversity $(\pi)$, number of polymorphic sites, number of haplotypes, and number of synonymous and nonsynonymous substitutions were obtained using the programs MEGA 5.05 and DnaSP 5.0 (Librado and Rozas, 2009). We also calculated Tajima's and Fu and Li's neutrality statistics using DnaSP 5.0.

\section{Phylogenetic analysis}

To reconstruct phylogenetic relationships between the cytochrome $b$ gene haplotypes, a neighbor-joining (NJ) method was used as implemented in MEGA 5.05 (with the Kimura 2-parameter model). Maximum likelihood (ML) analysis was performed using PHYML v.3.0 (Guindon and Gascuel, 2003) with the $\mathrm{HKY}+\mathrm{G}$ model, which is the best-fitting model that is selected by hierarchical likelihood ratio tests (hLRTs) in jModelTest program (Posada, 2008). The confidence of branches in NJ and ML trees was assessed using non-parametric bootstrapping searches of 1000 replicates.

Bayesian inference (BI) of phylogeny using the Markov Chain Monte Carlo technique (MCMC) was computed using BEAST 1.6.1 (Drummond and Rambaut, 2007). The $\mathrm{HKY}+\mathrm{G}$ model was selected as the best-fitting model of DNA substitution for mitochondrial DNA (mtDNA) segments that were studied using the Bayesian information criterion (BIC) as implemented in MEGA 5.05. Three independent analyses with Markov chains were simulated over 30,000,000 generations. The haplotype trees were visualized by FigTree 1.1.2. Bayesian posterior probabilities (BPP) that were $95 \%$ or greater were considered to be significant support (Leache and Reeder, 2002). As outgroups for the subgenus Martes mtDNAs, we used nucleotide sequences of the yellow-throated marten (Martes flavigula, GenBank accession No. HM106326), wolverine (Gulo gulo, AB051245), fisher (Martes pennanti, AF057131), and American mink (Mustela vison, AF057129). 


\section{Testing for the signatures of selection and molecular adaptation}

The ratios of the number of nonsynonymous substitutions per nonsynonymous site (Ka or $\mathrm{dN}$ ) to the number of synonymous substitutions per synonymous site (Ks or dS) between DNA sequences were calculated (Nei and Gojobori, 1986) using DnaSP 5.0. A Ka/Ks ratio that is significantly less than 1 indicates negative (purifying) selective pressure, and a ratio that is significantly greater than 1 indicates positive selection (Nielsen, 2001). The Ka/ Ks values were calculated for interspecific mtDNA divergence (true martens species versus M. flavigula), and the $\mathrm{Pi}(\mathrm{a}) / \mathrm{Pi}(\mathrm{s})$ values were calculated for intraspecific mtDNA comparisons using DnaSP 5.0.

A codon-based Z-test of purifying selection for analysis averaging over all mtDNA sequence pairs within species was used to test for a significant difference between $\mathrm{dN}$ and $\mathrm{dS}$ estimates, and the variance of the difference $(\mathrm{dS}-\mathrm{dN})$ was computed using the bootstrap method (1000 replicates) in MEGA 5.05 (Li et al., 1985). ML analysis of natural selection for each codon using the test statistic (dS-dN) was also conducted in MEGA 5.05 using the HyPhy software package (Kosakovsky et al., 2005).

Here, we focus also on amino acid differences that correspond to radical physicochemical variation (positive-destabilizing selection) and are expected to be linked to significant changes in function at the protein level (McClellan and McCracken, 2001; McClellan et al., 2005). Significant physicochemical amino acid changes among residues in cytochrome $b$ were identified by the algorithm that was implemented in TreeSAAP 3.2 (Woolley et al., 2003). TreeSAAP categorizes each amino acid site by positively and negatively destabilizing the site using 31 properties (henceforth, amino acid positions will be called sites). The cytochrome $b$ domain organization was taken from McClellan et al. (2005). To detect strong directional selective pressure, we considered only those changes that corresponded to category 8 (the most radical property changes category) at $\mathrm{P} \leq 0.001$ level (z-scores $>3.09$ ). TreeSAAP analysis was performed in subfamily Martinae based on the NJ tree of the complete cytochrome $b$ gene sequences.

\section{RESULTS}

\section{Cytochrome $b$ gene variability and phylogenetic analysis}

After mtDNA sequencing, 25 cytochrome $b$ gene haplotypes that were defined by 53 variable sites were revealed in 49 sables from Russia and China. In contrast, only 4 haplotypes that were defined by 5 variable sites were present in 28 pine martens from Denmark and Sweden. In 10 stone martens from Denmark, 3 haplotypes that were defined by 9 variable sites were found. All of the above haplotypes were respectively deposited in GenBank under accession Nos. HQ386861-HQ386885 for M. zibellina, HQ386886-HQ386888 for M. foina, and HQ386889-HQ386892 for M. martes. We should note also that the sable haplotypes that were deposited in GenBank under accession Nos. HQ386862 and HQ386864-HQ386885 were previously reported elsewhere (Li et al., 2013).

Table 2 summarizes information concerning the molecular diversity and neutrality tests of the studied mtDNA samples. We did not detect identical haplotypes between the sables and pine martens besides the sable haplotype Mz4 found in Daxing'anling Mountains in China 
and Primorye region (Lazo raion) in Russia, which is identical to a previously reported sequence (AF448241) of the pine marten from Sweden (Stone and Cook, 2002).

Table 2. Diversity indices and neutrality tests for cytochrome $b$ gene in species studied.

\begin{tabular}{|c|c|c|c|c|c|}
\hline \multirow[t]{3}{*}{ Genetic characteristics } & \multicolumn{5}{|c|}{ Species } \\
\hline & \multicolumn{3}{|c|}{ Martes zibellina } & \multirow[t]{2}{*}{ Martes martes } & \multirow[t]{2}{*}{ Martes foina } \\
\hline & Clade A & Clade B & Overall & & \\
\hline Sample size $(\mathrm{N})$ & 20 & 36 & 56 & 29 & 11 \\
\hline Number of haplotypes $(k)$ & 15 & 16 & 31 & 5 & 4 \\
\hline Number of polymorphic sites $(s)$ & 26 & 25 & 59 & 6 & 35 \\
\hline Haplotype diversity $(h)$ & $0.97 \pm 0.03$ & $0.94 \pm 0.02$ & $0.97 \pm 0.01$ & $0.67 \pm 0.05$ & $0.78 \pm 0.08$ \\
\hline Average number of pairwise differences $(i)$ & 3.97 & 4.74 & 9.98 & 1.47 & 9.06 \\
\hline Nucleotide diversity $(\pi)$ & 0.0035 & 0.0042 & 0.0088 & 0.0013 & 0.0079 \\
\hline D (Tajima's neutrality test) & $-1.78(\mathrm{P}>0.05)$ & $-0.74(\mathrm{P}>0.1)$ & $-0.77(\mathrm{P}>0.1)$ & $-0.103(\mathrm{P}>0.1)$ & $-1.13(\mathrm{P}>0.1)$ \\
\hline $\mathrm{F}^{*}(\mathrm{Fu}, \mathrm{Li}$ 's neutrality test) & $-2.39(\mathrm{P}>0.05)$ & $-1.05(\mathrm{P}>0.1)$ & $-2.501(\mathrm{P}<0.05)$ & $0.448(\mathrm{P}>0.1)$ & $-1.57(\mathrm{P}>0.1)$ \\
\hline Number of synonymous changes & 18 & 19 & 44 & 5 & 30 \\
\hline Number of nonsynonymous changes & 8 & 6 & 15 & 1 & 5 \\
\hline $\mathrm{Pi}(\mathrm{a}) / \mathrm{Pi}(\mathrm{s})$ & 0.121 & 0.1 & 0.082 & 0.034 & 0.048 \\
\hline $\begin{array}{l}\text { Z-test of selection: P value in favor of the } \\
\mathrm{dN}<\mathrm{dS} \text { hypothesis }\end{array}$ & $1.6^{*} 10^{-3}$ & $4.4 * 10^{-4}$ & $3.0^{*} 10^{-5}$ & $2.9 * 10^{-2}$ & $2.8 * 10^{-6}$ \\
\hline
\end{tabular}

The highest levels of mtDNA diversity were found in the sable and the stone marten, while the haplotype and nucleotide diversity of the pine marten was much lower (Table 2). The interspecific differences were minimal between the sable and the pine marten (divergence Dxy $=2.45 \%$ ), whereas differences between the pairs M. foina-M. zibellina and M. foina-M. martes were more pronounced, 8.9 and $7.4 \%$, respectively.

Using additional cytochrome $b$ gene sequences from GenBank, we analyzed phylogenetic relationships between species of true martens (Figure 1). Bayesian analysis (as well as $\mathrm{NJ}$ and ML results that are not shown here) revealed a strong structured topology of the tree with highly supported groupings at the interspecific level. In agreement with the results of other recent studies (Stone and Cook, 2002; Koepfli et al., 2008), our phylogeny indicates that true martens (subgenus Martes) are monophyletic and sister to the yellow-throated marten (M. flavigula). Within the subgenus Martes, Bayesian analysis demonstrated that M. americana was sister to the clade $[M$. melampus (M. martes + M. zibellina $)]$. The same result was attained in the maximum parsimony (MP) tree of the cytochrome $b$ gene (Stone and Cook, 2002), whereas the relationships between M. americana, M. melampus, and (M. martes $+M$. zibellina) were unresolved in the Bayesian tree that was based on the data matrix comprising 22 gene segments ( 12,000 base pairs) (Koepfli et al., 2008).

In the sable, 2 major clades-A (BPP: 1.0) and B (BPP: 0.99)-were revealed. Clade B was more diverse than clade $\mathrm{A}$ after taking into consideration such parameters as the average mean of pairwise nucleotide differences and nucleotide diversity (Table 2). In addition, within clade B, there is subclade B1, which had a highly significant BPP that was equal to 0.99.

\section{Testing for the signatures of selection and molecular adaptation}

Statistical tests of the neutrality of mtDNA cytochrome $b$ mutations have shown that negative (purifying) selection appears to be characteristic for the mitochondrial genome of the studied species (Table 3). Very low values of nonsynonymous to synonymous rate ratios 
(much less than 1.0) were found among mtDNA haplotypes in intra- and interspecific comparisons (Table 2, Table 3). Negative selection was detected with highly significant support using the Z-test of selection that was proposed by Li et al. (1985).

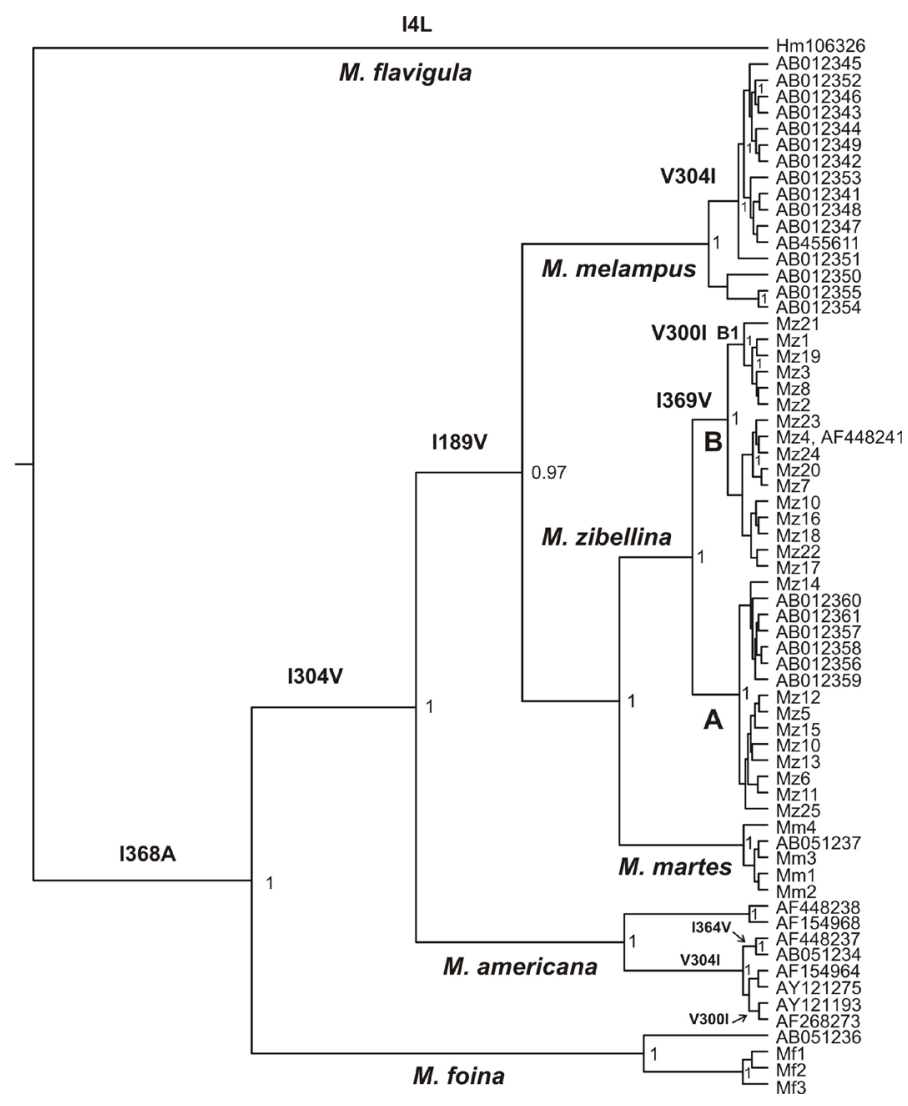

Figure 1. Bayesian inference of the phylogeny of the subgenus Martes under the $\mathrm{HKY}+\mathrm{G}$ model of nucleotide substitutions that was used to analyze cytochrome $b$ gene sequences. Wolverine, fisher, and American mink were also used as outgroups (not shown on the tree). Positions of clades A, B, and B1 of the sable are marked on the tree. On the branches, the radical amino acid changes that were revealed by TreeSAAP are shown.

Table 3. Tests of purifying selection for analysis averaging over all mtDNA haplotype pairs within species (Z-test and $\mathrm{Pi}(\mathrm{a}) / \mathrm{Pi}(\mathrm{s})$ values) and between species $(\mathrm{Ka} / \mathrm{Ks}$ values) in true martens.

\begin{tabular}{lcccr}
\hline Species & \multicolumn{2}{c}{ Z-test of selection } & Pi(a)/Pi(s) & Ka/Ks \\
\cline { 2 - 4 } & $\mathrm{dS}-\mathrm{dN}$ & $\mathrm{P}$ value & & \\
\hline Martes zibellina & 4.55 & $6.6^{*} 10^{-6}$ & 0.084 & 0.043 \\
M. martes & 2.22 & $1.4^{*} 10^{-2}$ & 0.066 & 0.044 \\
M. melampus & 4.42 & $1.1^{*} 10^{-5}$ & 0.067 & 0.047 \\
M. americana & 4.83 & $2.0^{*} 10^{-6}$ & 0.077 & 0.034 \\
M. foina & 5.0 & $9.8^{*} 10^{-7}$ & 0.051 & 0.039 \\
True martens & 10.49 & 0 & 0.035 & 0.042 \\
\hline
\end{tabular}

$\mathrm{Ka} / \mathrm{Ks}$ values were calculated for true martens mtDNA haplotypes in comparison with $M$. flavigula one. 
Meanwhile, purifying selection can be accompanied by positive selection at particular sites, leading to adaptive evolution. The test statistic (dS-dN) that was produced by ML analysis of selection for each codon found no sites in the true martens that were affected by positive selection. Meanwhile, significant physicochemical amino acid changes among residues in cytochrome $b$ were identified by the algorithm that was implemented in TreeSAAP, which compares the observed distribution of physicochemical changes that are inferred from a phylogenetic tree with an expected distribution based on the assumption of completely random amino acid replacement that is expected under the conditions of selective neutrality (Woolley et al., 2003; McClellan et al., 2005). As a result, 7 amino acid sites that were influenced by strong positive selection (with changes corresponding to the most radical property changes, category 8 , at the $\mathrm{P} \leq 0.001$ level) were detected by TreeSAAP (Figure 1). These sites were mainly located in the transmembrane domains: residue 189 in the D-helix, residues 300 and 304 in the F-helix, and residues 364, 368, and 369 in the H-helix according to the secondary amino acid structure of cytochrome $b$ (McClellan et al., 2005). Only site 4 in the M. flavigula branch was an amino acid residue where positively selected radical changes took place in the matrix domain in the N-terminal region of cytochrome $b$. It is also noteworthy that the majority of amino acid changes were detected to be isoleucine to valine and vice versa. TreeSAAP indicated that amino acid substitutions that were revealed in the cytochrome $b$ of true martens resulted in radical shifts in only 1 amino acid property, the equilibrium constant for the ionization of $\mathrm{COOH}\left(\mathrm{K}^{\prime}\right)$.

As we can see, radical changes accumulate sequentially during the process of cladogenesis (Figure 1). True martens are characterized by the radical change Ile368Ala, while subsequent taxonomic division is accompanied by radical substitutions Ile304Val and Ile189Val. Positive selection operates also in the M. zibellina branch. In the sable, the Ile369Val substitution is characteristic for clade B animals, and Val300Ile distinguishes the sables from subclade B1 (Figure 1). However, molecular adaptation was not detected in the pine marten or the stone marten.

\section{DISCUSSION}

It is well known that strong negative (purifying) selection plays a central role in the evolution of mtDNA to keep its important functions in energy metabolism (Stewart et al., 2008; Shen et al., 2010; Sun et al., 2011). Meanwhile, because negative selection cannot generate "improved" genes that affect the protein phenotype by itself, positive selection is the major mechanism of mtDNA adaptive evolution, taking into account the lack of recombination in animal mitochondria (Kimura, 1983; Shen et al., 2010). However, positive selection affects only a small fraction of sites, especially in mitochondrial genomes, and is much rarer than negative selection. In general, after a positive selection-driven amino acid replacement is over, positive selection transforms into negative selection (Bazykin and Kondrashov, 2011). Because cytochrome $b$ (and other mitochondrially encoded proteins) is an extremely conserved protein, thus reflecting its fundamental role in energy production, it is difficult to find evidence of positive selection using standard approaches that are based on nonsynonymous to synonymous rate ratios (McClellan et al., 2005; Zhang et al., 2005; Bazykin and Kondrashov, 2011). However, insight into the evolution of this protein can be obtained by inspecting amino 
acid substitutions with the software TreeSAAP, studying molecular adaptation as a function of changes in protein phenotype that result from corresponding changes in suites of physicochemical amino acid properties (McClellan et al., 2005). In this study, we detected such amino acid sites that were influenced by positive selection during speciation in the subgenus Martes (Figure 1).

TreeSAAP analysis showed that most of such amino acid sites were located in the transmembrane domains, and the majority of amino acid changes occurred from isoleucine to valine and vice versa. This is consistent with previous studies, which suggested that many of the amino acid replacements within the transmembrane segments include hydrophobic residues, especially leucine, isoleucine, and valine (Irwin et al., 1991). Although it is proposed that radical changes of amino acids in the transmembrane domains have been involved in the proton-input function (McClellan et al., 2005), we do not have enough evidence to suggest that mutations occurring in Martes cytochrome $b$ have functional implications, which were discovered in other mammals (McClellan and McCracken, 2001; Fink et al., 2004; McClellan et al., 2005; da Fonseca et al., 2008).

Amino acid substitutions that were detected in our study were found to be affected by positive-destabilizing selection for only 1 amino acid property, $\mathrm{K}^{\prime}$ (equilibrium constant for the ionization of $\mathrm{COOH}$ ). However, it is proposed that an increased $\mathrm{K}^{\prime}$ would make the cytochrome $b$ region more water-soluble and hydrophilic, and this may be of great importance regarding reduced reactive oxygen species (ROS) production (Beckstead et al., 2009). In addition, we suggest that the radical changes at these cytochrome $b$ residues in true martens may be the result of molecular adaptation to environmental conditions that are associated with species distribution. For example, M. zibellina populates mainly the northern forest, and it extends southward to $42^{\circ}$ in the mountainous areas of eastern Asia (Buskirk et al., 1996). This species requires a stronger ability to adjust its body temperature in winter than more southerndistributed marten species, such as M. flavigula. In addition, although it has similar pelts, the sable is considered better adapted to cold temperatures than the pine marten (Davison et al., 2001). Therefore, our results revealed that more abundant natural selection on the cytochrome $b$ protein among the sables, in comparison with the pine marten, is consistent with the above consideration. For both species, however, mtDNA studies suggest that Pleistocene climatic conditions played an important role in the phylogeographic differentiation of the pine marten and the sable as well as their phylogenetic groups (Ruiz-Gonzalez, 2011; Li et al., 2013). Further studies are required to describe processes of possible coevolution of genes and natural habitats of Martes species.

\section{ACKNOWLEDGMENTS}

Research supported by the Fundamental Research Funds for Central Universities (grant \#DL13CA12) and the Wildlife Conservation and Natural Reserve Construction Project of the State Forestry Administration of China. We are grateful to the following people for supplying tissue samples: Prof. Wei Zhang, Yanchun Xu, and Guangcai Sun at the College of Wildlife Resources, Northeast Forestry University and Dr. Aleksey I. Antonov, Senior Research Fellow at Khingan State Nature Reserve, Russia. We thank Tom Dahmer for editing early versions of this manuscript. 


\section{REFERENCES}

Anderson E (1970). Quaternary evolution of the genus Martes (Carnivora, Mustelidae). Acta Zool. Fenn. 130: 127-132.

Bazykin GA and Kondrashov AS (2011). Detecting past positive selection through ongoing negative selection. Genome Biol. Evol. 3: 1006-1013.

Beckstead WA, Ebbert MT, Rowe MJ and McClellan DA (2009). Evolutionary pressure on mitochondrial cytochrome $b$ is consistent with a role of CytbI7T affecting longevity during caloric restriction. PLoS One 4: e5836.

Buskirk SW, Ma YQ, Xu L and Jiang ZW (1996). Winter habitat ecology of sables (Martes zibellina) in relation to forest management in China. Ecol. Appl. 6: 318-325.

da Fonseca RR, Johnson WE, O'Brien SJ, Ramos MJ, et al. (2008). The adaptive evolution of the mammalian mitochondrial genome. BMC Genomics 9: 119.

Davison A, Birks JD, Brookes RC, Messenger JE, et al. (2001). Mitochondrial phylogeography and population history of pine martens Martes martes compared with polecats Mustela putorius. Mol. Ecol. 10: 2479-2488.

Drummond AJ and Rambaut A (2007). BEAST: Bayesian evolutionary analysis by sampling trees. BMC Evol. Biol. 7: 214.

Fink S, Excoffier L and Heckel G (2004). Mitochondrial gene diversity in the common vole Microtus arvalis shaped by historical divergence and local adaptations. Mol. Ecol. 13: 3501-3514.

Foote AD, Morin PA, Durban JW, Pitman RL, et al. (2011). Positive selection on the killer whale mitogenome. Biol. Lett. 7: 116-118.

Gershoni M, Templeton AR and Mishmar D (2009). Mitochondrial bioenergetics as a major motive force of speciation. Bioessays 31: 642-650.

Guindon S and Gascuel O (2003). A simple, fast, and accurate algorithm to estimate large phylogenies by maximum likelihood. Syst. Biol. 52: 696-704.

Hosoda T, Suzuki H, Harada M, Tsuchiya K, et al. (2000). Evolutionary trends of the mitochondrial lineage differentiation in species of genera Martes and Mustela. Genes Genet. Syst. 75: 259-267.

Irwin DM, Kocher TD and Wilson AC (1991). Evolution of the cytochrome $b$ gene of mammals. J. Mol. Evol. 32: 128-144.

Kimura M (1983). The Neutral Theory of Molecular Evolution. Cambridge University Press, Cambridge.

Koepfli KP and Wayne RK (1998). Phylogenetic relationships of otters (Carnivora: Mustelidae) based on mitochondrial cytochrome $b$ sequences. J. Zool. 246: 401-416.

Koepfli KP, Deere KA, Slater GJ, Begg C, et al. (2008). Multigene phylogeny of the Mustelidae: resolving relationships, tempo and biogeographic history of a mammalian adaptive radiation. BMC Biol. 6: 10 .

Kosakovsky PSL, Frost SDW and Muse SV (2005). HyPhy: hypothesis testing using phylogenies. Bioinformatics 21: 676-679.

Kurose N, Masuda R, Siriaroonrat B and Yoshida MC (1999). Intraspecific variation of mitochondrial cytochrome $b$ gene sequences of the Japanese Martes melampus and the sable Martes zibellina (Mustelidae, Carnivora, Mammalia) in Japan. Zool. Sci. 16: 693-700.

Leache AD and Reeder TW (2002). Molecular systematics of the Eastern Fence Lizard (Sceloporus undulatus): a comparison of Parsimony, Likelihood, and Bayesian approaches. Syst. Biol. 51: 44-68.

Li B, Xu YC, Ma Y, Elmeros M, et al. (2011). A PCR-RFLP-based method to distinguish sable (Martes zibellina) and pine marten (Martes martes). Acta Theriol. 56: 283-288.

Li B, Malyarchuk B, Ma Z, Derenko M, et al. (2013). Phylogeography of sable (Martes zibellina L. 1758) in the southeast portion of its range based on mitochondrial DNA variation: highlighting the evolutionary history of the sable. Acta Theriol. 58: 139-148.

Li WH, Wu CI and Luo CC (1985). A new method for estimating synonymous and nonsynonymous rates of nucleotide substitution considering the relative likelihood of nucleotide and codon changes. Mol. Biol. Evol. 2: 150-174.

Librado P and Rozas J (2009). DnaSP v5: a software for comprehensive analysis of DNA polymorphism data. Bioinformatics 25: 1451-1452.

Malyarchuk B, Derenko M, Berman D, Perkova M, et al. (2010). Phylogeography and molecular adaptation of Siberian salamander Salamandrella keyserlingii based on mitochondrial DNA variation. Mol. Phylogenet. Evol. 56: 562-571.

McClellan DA and McCracken KG (2001). Estimating the influence of selection on the variable amino acid sites of the cytochrome $b$ protein functional domains. Mol. Biol. Evol. 18: 917-925.

McClellan DA, Palfreyman EJ, Smith MJ, Moss JL, et al. (2005). Physicochemical evolution and molecular adaptation of the cetacean and artiodactyl cytochrome $b$ proteins. Mol. Biol. Evol. 22: 437-455.

Nei $\mathrm{M}$ and Gojobori T (1986). Simple methods for estimating the numbers of synonymous and nonsynonymous nucleotide substitutions. Mol. Biol. Evol. 3: 418-426. 
Nielsen R (2001). Statistical tests of selective neutrality in the age of genomics. Heredity 86: 641-647.

Posada D (2008). jModelTest: phylogenetic model averaging. Mol. Biol. Evol. 25: 1253-1256.

Ruiz-Gonzalez A (2011). Phylogeography and Non-Invasive Landscape Genetics of the European Pine Marten (Martes martes L. 1758): Insights into Ancient and Contemporary Processes Shaping Genetic Variation. Universidad del Pais Vasco, Vitoria-Gasteiz.

Shen YY, Liang L, Zhu ZH, Zhou WP, et al. (2010). Adaptive evolution of energy metabolism genes and the origin of flight in bats. Proc. Natl. Acad. Sci. U. S. A. 107: 8666-8671.

Stewart JB, Freyer C, Elson JL, Wredenberg A, et al. (2008). Strong purifying selection in transmission of mammalian mitochondrial DNA. PLoS Biol. 6: e10.

Stone KD and Cook JA (2002). Molecular evolution of Holarctic martens (genus Martes, Mammalia: Carnivora: Mustelidae). Mol. Phylogenet. Evol. 24: 169-179.

Stone KD, Flynn RW and Cook JA (2002). Post-glacial colonization of northwestern North America by the forestassociated American marten (Martes americana, Mammalia: Carnivora: Mustelidae). Mol. Ecol. 11: 2049-2063.

Sun YB, Shen YY, Irwin DM and Zhang YP (2011). Evaluating the roles of energetic functional constraints on teleost mitochondrial-encoded protein evolution. Mol. Biol. Evol. 28: 39-44.

Tamura K, Peterson D, Peterson N, Stecher G, et al. (2011). MEGA5: molecular evolutionary genetics analysis using maximum likelihood, evolutionary distance, and maximum parsimony methods. Mol. Biol. Evol. 28: 2731-2739.

Trumpower BL (1990). The protonmotive Q cycle. Energy transduction by coupling of proton translocation to electron transfer by the cytochrome $b c_{1}$ complex. J. Biol. Chem. 265: 11409-11412.

Woolley S, Johnson J, Smith MJ, Crandall KA, et al. (2003). TreeSAAP: selection on amino acid properties using phylogenetic trees. Bioinformatics 19: 671-672.

Yu L, Peng D, Liu J, Luan P, et al. (2011). On the phylogeny of Mustelidae subfamilies: analysis of seventeen nuclear non-coding loci and mitochondrial complete genomes. BMC Evol. Biol. 11: 92.

Zhang J, Nielsen R and Yang Z (2005). Evaluation of an improved branch-site likelihood method for detecting positive selection at the molecular level. Mol. Biol. Evol. 22: 2472-2479. 